\title{
Delayed Onset Abducens Nerve Palsy following Uncomplicated Large Cystic Vestibular Schwannoma Resection: Case Report
}

\author{
Sima Sayyahmelli ${ }^{1}$ Pinar Aydin ${ }^{1} \quad$ Mustafa K. Baskaya ${ }^{1}$ \\ ${ }^{1}$ Department of Neurological Surgery, University of Wisconsin \\ Medical School, Madison, Wisconsin, United States \\ Address for correspondence Mustafa K. Baskaya, MD, Department of \\ Neurological Surgery, University of Wisconsin Medical School, K4/834 \\ CSC, 600 Highland Avenue, Madison, WI 53792-0001, United States \\ J Neurol Surg Rep 2019;80:e37-e40. \\ (e-mail: baskaya@neurosurgery.wisc.edu).
}

\author{
Abstract \\ Keywords \\ - abducens nerve \\ - acoustic canal \\ - delayed onset \\ - vestibular \\ schwannoma
}

\begin{abstract}
Although delayed facial palsy after vestibular schwannoma (VS) surgery is a poorly understood but a well-known phenomenon, other delayed cranial nerve palsies in the cerebellopontine angle have not been reported after VS surgery. In this report, we describe a 54-year-old woman with a large cystic VS who experienced double vision and a new delayed onset right abducens nerve (AbN) palsy, 3 weeks after gross total resection of VS via a translabyrinthine approach. To the best of our knowledge, this is the first report describing delayed isolated AbN palsy after uncomplicated VS surgery. Magnetic resonance imaging findings and the management of this complication following VS surgery are discussed in this case report.
\end{abstract}

\section{Introduction}

Large vestibular schwannoma (VS) can cause considerable brain stem and adjacent cranial nerves compression that increases surgery-related morbidity and mortality rates. ${ }^{1}$ Delayed facial palsy (DFP) is a well-known phenomenon that has been reported to occur in 15 to $29 \%$ of patients undergoing VS resections. ${ }^{2}$ However, the exact etiology and mechanism of DFP remain unknown. ${ }^{3}$

In spite of being very rare, abducens nerve (AbN) palsy can occur as an early onset and transient complication after VS surgery and carries significant morbidity. ${ }^{1}$

We describe a 54-year-old woman with a large cystic VS, who experienced double vision and delayed onset right $\mathrm{AbN}$ palsy 3 weeks following translabyrinthine resection surgery. To the best of our knowledge, this is the first report describing delayed isolated $\mathrm{AbN}$ palsy after uncomplicated VS surgery.

\section{Case Report}

A 54-year-old woman with a 1-year history of imbalance, dizziness, and difficulty in word discrimination presented in the neurosurgery clinic. Magnetic resonance imaging (MRI) showed an approximately $6 \mathrm{~cm}$, large cystic right cerebellopontine angle mass with extension into the internal acoustic canal. There was also a significant amount of brain stem and fourth ventricle compression with early signs of hydrocephalus (-Fig. 1A, B).

The patient underwent gross total resection using a translabyrinthine approach. The surgery was uneventful. Postoperative MRI confirmed gross total resection ( - Fig. 2A, B). Per the routine protocol, she received steroids and valacyclovir for 10 days in the postoperative period. Her facial nerve function was House-Brackmann grade II immediately after the surgery. Other cranial nerves, except right-sided hearing loss which existed before surgery, were intact, and she did not experience any subjective double vision after surgery.

Three weeks after surgery, her facial nerve function had improved to House-Brackmann grade I. However, she reported having double vision and had a new AbN palsy on the right side. Her other cranial nerve functions remained intact except hearing loss on the right side, which had existed before surgery. MRI showed a persistent edema within the right middle cerebellar peduncle that was present in both the pre- and immediate postoperative MRIs (- Fig. 3A, B). MRI received

November 30, 2018 accepted after revision June 18, 2019
DOI https://doi.org/

10.1055/s-0039-1694737. ISSN 2193-6358.
๑) 2019 Georg Thieme Verlag KG
Stuttgart · New York

License terms

(1) $\circledast \circledast$ 


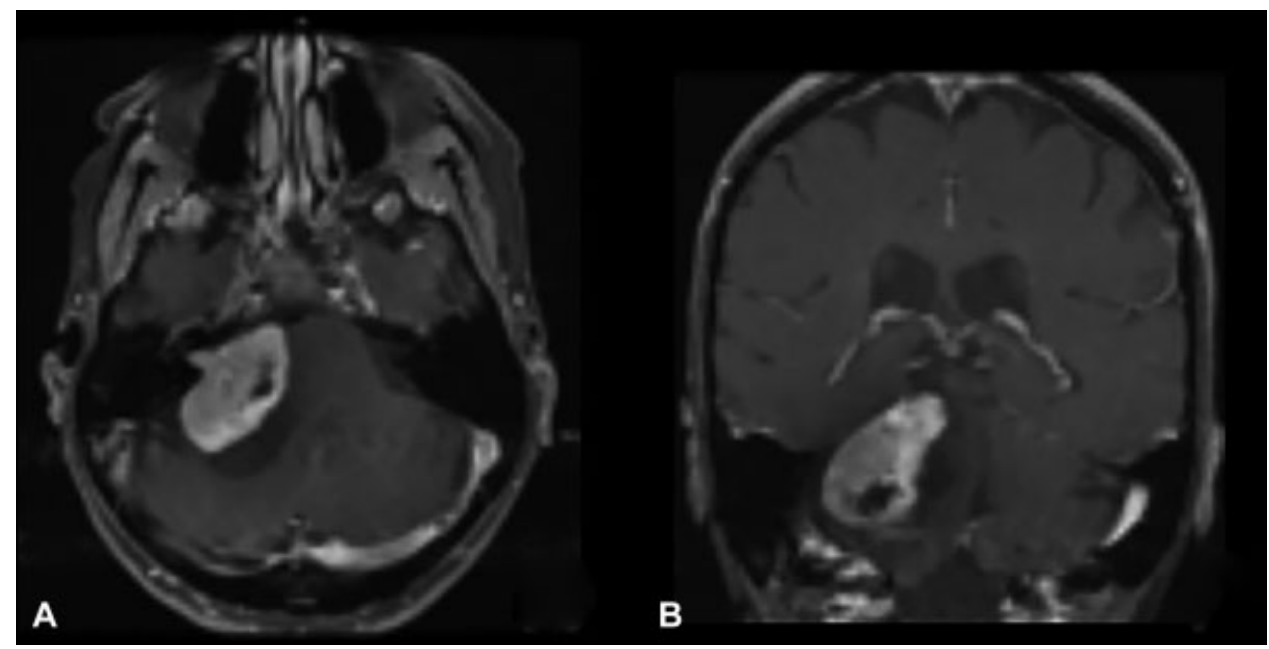

Fig. 1 Preoperative post-contrast axial (A) and coronal (B) T1-weighted MRIs show an approximatyely $6 \mathrm{~cm}$, large cystic right cerebellopontine angle mass with extension into the internal acoustic canal. Note brain stem and fourth ventricle compression with the early signs of hydrocephalus. MRI, magnetic resonance imaging.

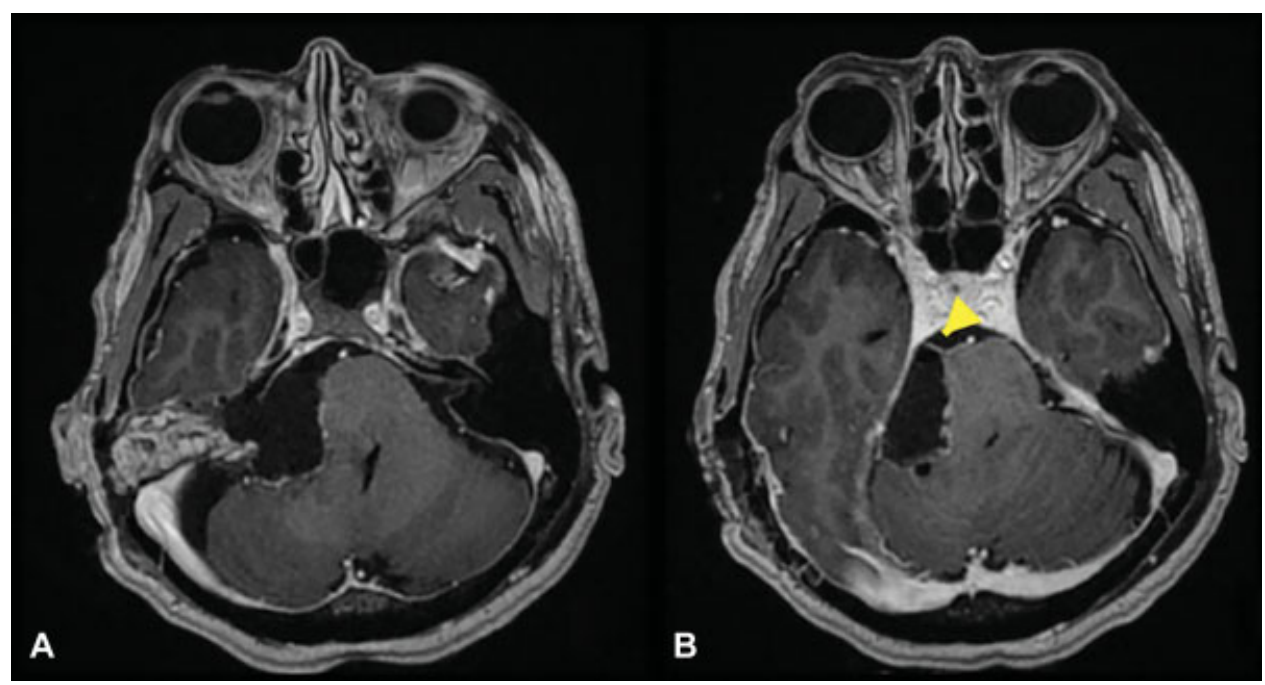

Fig. 2 Postoperative postcontrast axial (A, B) T1-weighted MRIs confirmed gross total resection without any enlargement or abnormal enhancement of the right trigeminal nerve and $\mathrm{AbN}$ (yellow arrowhead). AbN, abducens nerve; MRI, magnetic resonance imaging.

also showed a mild abnormal enhancement of the right trigeminal nerve and $\mathrm{AbN}$ ( - Fig. 3C, D). Oral dexamethasone and valacyclovir were then restarted for an additional 2 weeks. Her reported double vision at 3 weeks after surgery and delayed onset $\mathrm{AbN}$ palsy on the right side ( - Fig. 4A) were completely improved ( - Fig. 4B). At a 3-year follow-up, the patient remained tumor free with normal cranial nerve function, except for her prior right-sided hearing loss.

\section{Discussion}

DFP generally occurs between 4 and 30 days after VS surgery ${ }^{4}$ where there is otherwise complete or near-complete recovery of facial nerve function. ${ }^{3}$ Since the role of viral reactivation as a cause of some portions of DFP was elucidated, some studies ${ }^{5}$ have advocated for the use of perioperative famciclovir, which has been reported to reduce the incidence of DFP from $25 \%$ in the control group to $20 \%$.
In addition to the risk of injury to the seventh and eighth cranial nerves, other cranial nerves deficits may also occur in the patients with a large VSs. Immediate AbN palsy was present in $4 \%$ of a series of giant VSs resections published by Samii et al. ${ }^{1}$ However, delayed AbN palsy after VS surgery has not been reported previously. To the best of our knowledge, this is the first report describing delayed isolated AbN palsy after uncomplicated VS surgery, although there is a case report of delayed isolated AbN palsy following uncomplicated microvascular decompression for hemifacial spasm. ${ }^{6}$ Further research is needed to clarify the etiology of these delayed cranial nerve palsies.

The correlation between the postoperative enhancement of the cranial nerves and the onset of new cranial nerve deficits has not been evaluated in the literature. In the case presented here, a slight contrast enhancement of the $\mathrm{AbN}$ and the trigeminal nerves, with enlargement of both nerves, was noted on the postoperative 3-week MRI ( $\mathbf{F i g . ~ 3 C , ~ D ) . ~ A l t h o u g h ~ i t ~ i s ~}$ 


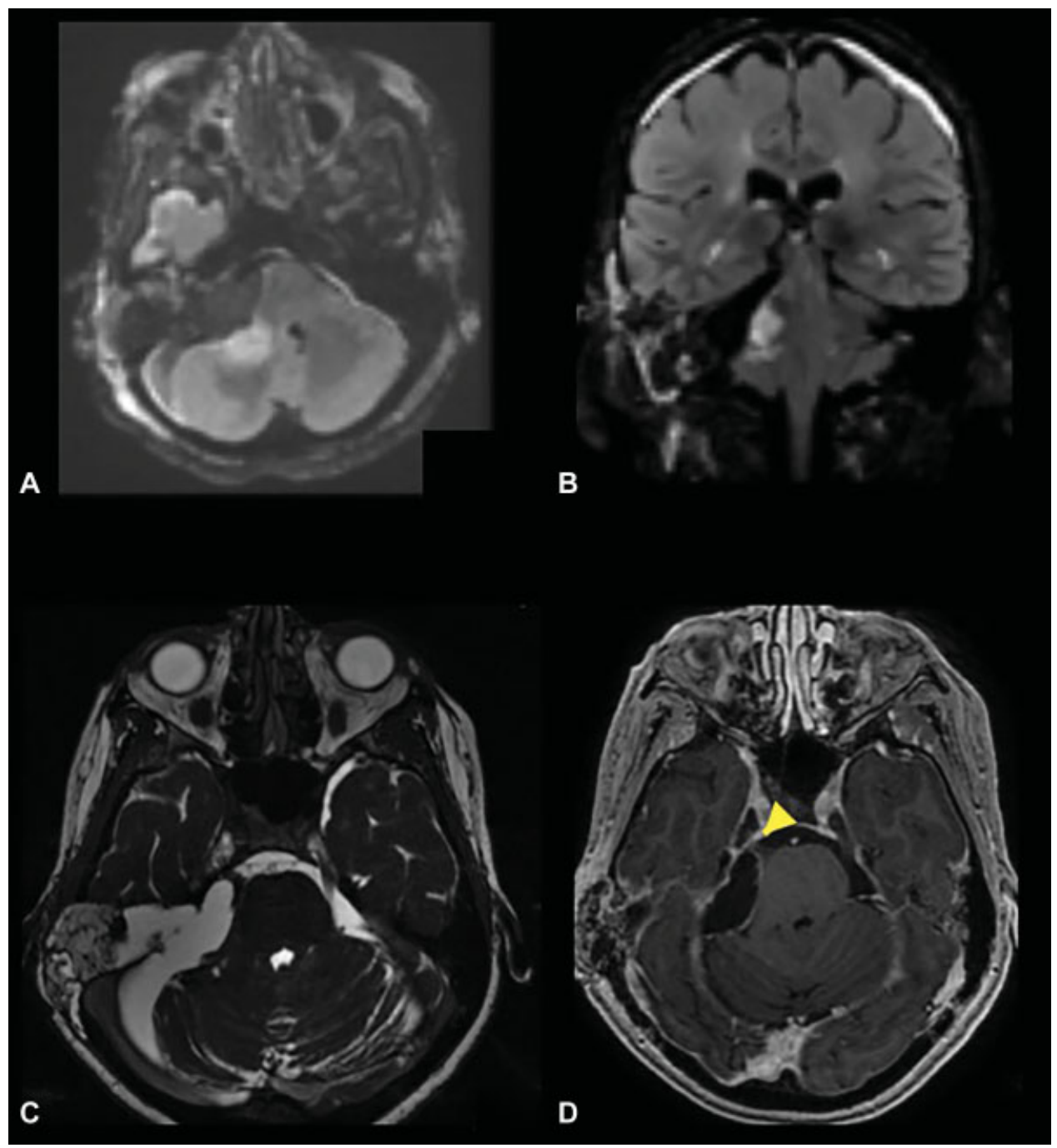

Fig. 3 Postoperative postcontrast axial (A) and coronal (B) T1-weighted MRIs show persistent edema within the right middle cerebellar peduncle and mild enlargement (C) and abnormal enhancement (D) of the right trigeminal nerve and AbN (yellow arrowhead). AbN, abducens nerve; MRI, magnetic resonance imaging.

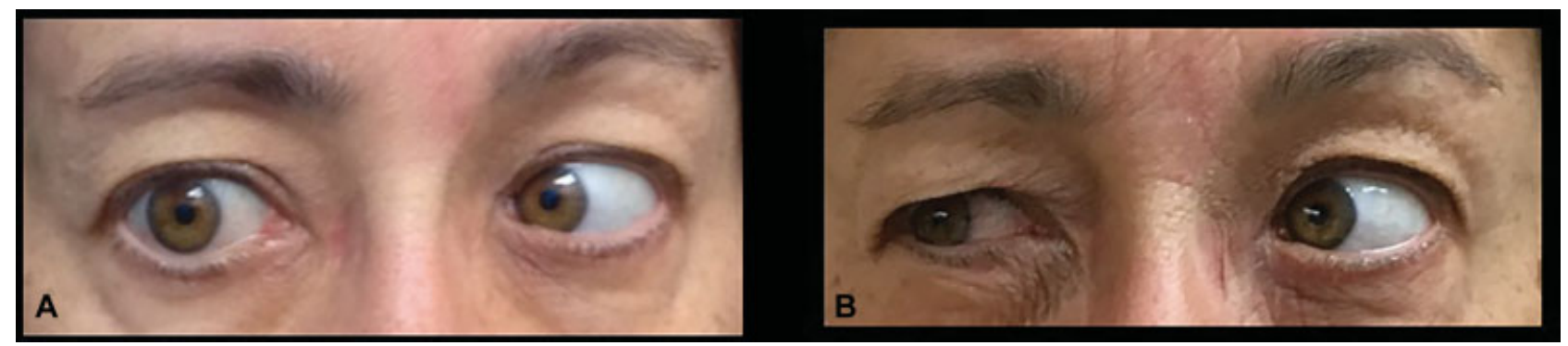

Fig. 4 Three weeks after surgery, the examination of the extraocular eye movements showed a new-onset AbN palsy on the right side (A). At 3year follow-up, the patient remains without recurrence of VS and the examination of the extraocular eye movements showed completely improvement of $A b N$ function (B). AbN, abducens nerve; VS, vestibular schwannoma.

difficult to elucidate the clinical significance of these radiological findings, these observations were only correlated with the $\mathrm{AbN}$ and not with the trigeminal nerve. Vulnerability of the $\mathrm{AbN}$ has been attributed to the angulation points of the nerve and not to its long intracranial course. ${ }^{7}$

We hypothesize that asymptomatic trigeminal nerve involvement can be attributed to different symptomatic thresholds between the trigeminal nerve and the $\mathrm{AbN}$ for the same pathologic process. It is also possible that subtle facial sensory deficits related to trigeminal nerve involvement could be present, which could not be elucidated by neurological examination.

\section{Conclusion}

Delayed isolated AbN palsy following uncomplicated VS surgery is an extremely rare complication, and to the best of our 
knowledge, this is the first report describing this complication. Although the etiology and the best management options for DFP are still unknown, surgeons should keep the possibility of delayed isolated AbN palsy after uncomplicated VS surgery in mind. Steroids along with valacyclovir, as recommended in DFP, might be used to treat delayed AbN palsy after VS surgery.

\section{Disclosure of Funding}

None.

\section{Conflict of Interest}

None.

\section{References}

1 Samii M, Gerganov VM, Samii A. Functional outcome after complete surgical removal of giant vestibular schwannomas. J Neurosurg 2010;112(04):860-867
2 Sampath P, Rhines LD, Holliday MJ, Brem H, Long DM. Late-onset facial nerve degeneration after vestibular schwannoma surgery: incidence, putative mechanisms, and prevention. Neurosurg Focus 1998;5(03): e6

3 Morton RP, Ackerman PD, Pisansky MT, et al. Prognostic factors for the incidence and recovery of delayed facial nerve palsy after vestibular schwannoma resection. J Neurosurg 2011;114(02): 375-380

4 Grant GA, Rostomily RR, Kim DK, et al. Delayed facial palsy after resection of vestibular schwannoma. J Neurosurg 2002;97(01): 93-96

5 Brackmann DE, Fisher LM, Hansen M, Halim A, Slattery WH. The effect of famciclovir on delayed facial paralysis after acoustic tumor resection. Laryngoscope 2008;118(09):1617-1620

6 Han JS, Lee JA, Kong DS, Park K. Delayed cranial nerve palsy after microvascular decompression for hemifacial spasm. J Korean Neurosurg Soc 2012;52(04):288-292

7 Yaman ME, Ayberk G, Eylen A, Ozveren MF. Isolated abducens nerve palsy following lumbar puncture: case report and review of the mechanism of action. J Neurosurg Sci 2010;54(03):119-123 